\title{
A Pretargeted Imaging Strategy for EGFR Positive Colorectal Carcinoma via the Modulation of Tz- radioligand Pharmacokinetics
}

\section{Lin Qiu}

Zhongshan Hospital Fudan University

\section{Hui Tan}

Zhongshan Hospital Fudan University

Qingyu Lin

Zhongshan Hospital Fudan University

\section{Zhan Si}

Zhongshan Hospital Fudan University

Jun Zhou

Zhongshan Hospital Fudan University

Tingting Wang

Zhongshan Hospital Fudan University

\section{Dengfeng Cheng}

Zhongshan Hospital Fudan University

Hongcheng Shi ( $\nabla$ shihongcheng163@163.com )

Fudan University https://orcid.org/0000-0003-1922-1359

\section{Original research}

Keywords: Colorectal cancer; EGFR; Cetuximab; Pretargeted imaging; Click chemistry

Posted Date: February 28th, 2020

DOI: https://doi.org/10.21203/rs.2.24836/v1

License: (1) (1) This work is licensed under a Creative Commons Attribution 4.0 International License. Read Full License

Version of Record: A version of this preprint was published at Molecular Imaging and Biology on September 10th, 2020. See the published version at https://doi.org/10.1007/s11307-020-01539-z. 


\section{Abstract}

Objective: Previously, we successfully developed a 6-hydrazinonicotinic acid (HYNIC)-modified terazine (Tz) derivative (HYNIC-PEG 11 -Tz) and labeled with Technetium-99m ( $\left.{ }^{99 \mathrm{~m}} \mathrm{Tc}\right)$ with a high radiochemical

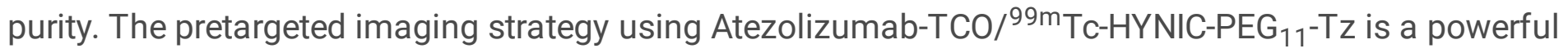
tool for evaluating Programmed Cell Death Ligand-1 (PD-L1) expression in xenograft mice tumor models. However, the surplus unclicked ${ }^{99 \mathrm{~m}} \mathrm{Tc}-\mathrm{HYNIC}-\mathrm{PEG}_{11}-\mathrm{Tz}$ is cleared somewhat sluggishly through the intestines. This is certainly not an ideal situation for imaging for abdominal tumors, especially for colorectal cancer. In order to shift the excretion of the Tz-radioligand to the renal system, we have sought to develop a novel Tz-radioligand by add a polypeptide linker between $\mathrm{HYNIC}$ and $\mathrm{PEG}_{11}$. Methods: A polypeptide-modified Tz (HYNIC-Polypeptide-PEG ${ }_{11}-\mathrm{Tz}$ ) was synthesized and radiolabeled with ${ }^{99 \mathrm{~m}} \mathrm{TC}$, and the Cetuximab was covalently modified with transcyclooctene (TCO-NHS). The stability of ${ }^{99 \mathrm{~m}} \mathrm{TC}-$ HYNIC-Polypeptide-PEG 11 -Tz was evaluated in vitro, and its blood pharmacokinetic test was performed in

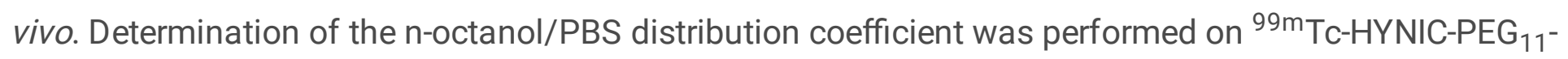
Tz and ${ }^{99 m}$ Tc-HYNIC-Polypeptide-PEG 11 -Tz to determine the effect of linker modification on their hydrophobicity. In vitro ligation reactivity of ${ }^{99 \mathrm{~m}} \mathrm{Tc}-\mathrm{HYNIC}$-Polypeptide-PEG $11^{-}$- $\mathrm{z}$ towards Cetuximab-TCO was tested. Pretargeted HCT116 cell immunoreactivity binding assay was evaluated. The biodistribution and imaging of ${ }^{99 \mathrm{~m}} \mathrm{Tc}-\mathrm{HYNIC}$-Polypeptide-PEG ${ }_{11}$-Tz was performed to observe the clear pathway of this novel Tz-radioligand. Pretargeted biodistribution of three different accumulation intervals $(24 \mathrm{~h}, 48 \mathrm{~h}$, and $72 \mathrm{~h}$ ) was performed to determine the optimal pretargeted interval time in nude mice bearing HCT116 tumor xenografts. Comparison of pretargeted (Cetuximab-TCO 48h/ ${ }^{99 \mathrm{~m}} \mathrm{Tc}_{\mathrm{HYNIC}-\mathrm{PEG}} \mathrm{H}^{-\mathrm{Tz}} 6 \mathrm{~h}$ ) and (Cetuximab-TCO 48h/ ${ }^{99 \mathrm{~m}} \mathrm{Tc}-\mathrm{HYNIC}-$ Polypeptide-PEG $11^{-T z} 6 \mathrm{~h}$ ) imaging was performed to show the effect of the two Tz-radioligands with different excretion pathway on tumor imaging. Results: HYNICPolypeptide-PEG ${ }_{11}-\mathrm{Tz}$ was successfully radiosynthesized with ${ }^{99 \mathrm{~m}} \mathrm{Tc}$, and a radiochemical purity greater than $95 \%$ were obtained as confirmed by radio high-performance liquid chromatography (HPLC). ${ }^{99 m}$ TcHYNIC-Polypeptide-PEG $11^{-}$Tz showed favorable stability in NS, PBS, and FBS and rapid blood clearance in mice. Liquid chromatograph-mass spectrometer (LC-MS) indicated the presence of an average 8.1 TCO moieties per Cetuximab. Size exclusion HPLC revealed almost complete reaction between Cetuximab-TCO and ${ }^{99 \mathrm{~m}} \mathrm{Tc}-\mathrm{HYNIC}$-Polypeptide- $\mathrm{PEG}_{11}$-Tz in vitro, with the 8:1 Tz-to-mAb reaction providing a conversion yield of $87.83 \pm 3.27 \%$. Pretargeted cell immunoreactivity binding assay showed high affinity to HCT116 cells. The biodistribution and imaging of ${ }^{99 \mathrm{~m}} \mathrm{Tc}$-HYNIC-Polypeptide-PEG ${ }_{11}$-Tz demonstrated that the Tzradioligand was cleared through kidneys. After allowing $24 \mathrm{~h}, 48 \mathrm{~h}$ and $72 \mathrm{~h}$ for accumulation of Cetuximab-TCO in HCT116 tumor, pretargeted biodistribution revealed an uptake of the radiotracer in the tumor with tumor-to-blood ratio of $0.83,1.40$, and 1.15 , respectively. Both pretargeted (Cetuximab-TCO 48h/ ${ }^{99 \mathrm{~m} T c-H Y N I C-P E G}{ }_{11}$-Tz 6h) and (Cetuximab-TCO 48h/ ${ }^{99 m}$ Tc-HYNIC-Polypeptide-PEG $11^{-T z} 6 \mathrm{~h}$ ) imaging delineated the HCT116 tumor clearly. However, pretargeted imaging strategy using CetuximabTCO 48h/ ${ }^{99 m}$ Tc-HYNIC-Polypeptide-PEG 11 -Tz $6 \mathrm{~h}$ ) could be used for diagnosing colorectal cancer since 
the surplus unclicked ${ }^{99 \mathrm{~m}} \mathrm{TC}-\mathrm{HYNIC}-$ Polypeptide-PEG 11 -Tz is cleared through urinary system and produces low abdominal uptake background. Conclusion: We developed a novel pretargeted imaging strategy (Cetuximab-TCO $/{ }^{99 \mathrm{~m}} \mathrm{Tc}$-HYNIC-Polypeptide-PEG 11 -Tz) for imaging colorectal cancer since the surplus unclicked ${ }^{99 \mathrm{~m}} \mathrm{Tc}$-HYNIC-Polypeptide-PEG $1{ }_{11}$-Tz produces low abdominal uptake background, which broadens the application scope of pretargeted imaging strategy.

\section{Introduction}

Colorectal cancer (CRC) is a common malignant tumor of the gastrointestinal tract with its morbidity ranking 3th among all malignant tumors and its mortality ranking 4th in the world, which poses a serious threat to human health [1]. In recent years, with the changes of lifestyle, the incidence of CRC has been increased year by year. Early diagnosis and treatment will greatly improve the prognosis of CRC, most of which could be cured in the early stage with a 5 -year survival rate of $90 \%$, while only $12.5 \%$ for advanced CRC [2]. Therefore, it is greatly significant for the diagnosis of CRC, especially for the early diagnosis, to research and develop the specific targeted molecular probe for CRC. At present, the clinical diagnosis for CRC mainly relies on endoscopy and endoscopy-guided biopsy, imaging methods such as computed tomography (CT), magnetic resonance imaging (MRI), and 2-deoxy-2- $\left[{ }^{18} \mathrm{~F}\right]$ fluoro-D-glucose $\left({ }^{18} \mathrm{~F}-\mathrm{FDG}\right)$ positron emission tomography/computed tomography (PET/CT). Invasive pathological biopsy guided by endoscopy is still the gold standard for clinical diagnosis of CRC. Common endoscopy mainly relies on morphological changes for the diagnosis of CRC, and it is easy to miss lesions with insignificant morphological changes, especially for early and micro lesions [3]. With the development of magnifying endoscopy, confocal laser endoscopy, and fluorescence endoscopy, endoscopic techniques could detect microscopic pathological changes and increase the diagnosis level of CRC. However, it is still difficult to detect surrounding tissue invasion and distant metastasis and achieve targeted imaging of CRC for these endoscopic methods. Anatomical imaging methods such as endoscopic ultrasonography, CT, or MRI have obvious advantages in the evaluation of surrounding tissue infiltration, lymph node and distant metastasis. But the imaging techniques are not specific for the diagnosis of CRC. As a functional imaging technology, ${ }^{18} \mathrm{~F}-\mathrm{FDG} \mathrm{PET} / \mathrm{CT}$ can reflect the tumor glucose metabolism. However, the most common positron radioactive tracer, ${ }^{18} \mathrm{~F}-\mathrm{FDG}$, is still not a specific targeting molecular probe for CRC.

Currently, targeted molecular probes for CRC mainly include antibody [4-5], polypeptide [7, 8], and nanoparticle $[9,10]$ probes for epithelial growth factor receptor (EGFR) or vascular endothelial growth factor (VEGF). Antibody probes have exquisite affinity and selectivity for molecular targets such as EGFR or VEGF overexpressed by CRC. However, due to the slow pharmacokinetics, the use of antibodies as tracers requires labeling with isotopes with long half-lives (e.g., ${ }^{111} \mathrm{In},{ }^{64} \mathrm{Cu}$, or ${ }^{131} \mathrm{I}$ ), which significantly increases the radiation dose to non-target tissues. Polypeptide probes have several advantages over antibody probes including low molecular weight, reduced circulation time, easy access to target sites, and passivity to the immune system with little or no immunogenicity. However, the stability and affinity to target sites vary among different polypeptide tracers. Nanoparticle probes are equipped with different 
targeting units for different receptors, but this type of probe poses a potential threat of immunogenicity and renal toxicity.

EGFR has become a therapeutic target for CRC due to the close association of the EGFR expression with disease progression and metastasis [11]. The therapeutic antibody Cetuximab have been approved by FDA for treating CRC. As EGFR inhibitor, Cetuximab specifically targets the extracellular domain of the EGFR and block the intracellular tyrosine kinase activity. Moreover, molecular tracer using Cetuximab labeled with the long-lived radionuclides (such as ${ }^{111} \mathrm{In},{ }^{89} \mathrm{Zr},{ }^{64} \mathrm{Cu}$, and ${ }^{124} \mathrm{I}$ ) has been developed to detect the EGFR expression and evaluate the therapeutic response to EGFR-blocking [12-16]. However, extended circulation times of radiolabeled antibodies creates prohibitively high radiation dose to healthy organs and low tumor/background imaging contrast. In this study, we will develop a novel pretargeted imaging strategy for evaluating EGFR expression of CRC.

Previously, we developed a pretargeted single photon emission computed tomography (SPECT) imaging strategy for evaluating immune checkpoint ligand PD-L1 expression in tumor based on bioorthogonal Diels-Alder click chemistry [17]. The molecular probe mainly includes two components: TCO-modified Atezolizumab (Atezolizumab-TCO) that could target PD-L1 and ${ }^{99 \mathrm{~m}}$ Tc labeled Tz-radioligand ${ }^{99 \mathrm{~m} T c-}$ HYNIC-PEG 11 -Tz). Pretargeted (Atezolizumab-TCO/ ${ }^{99 m} \mathrm{Tc}^{-H Y N I C-P E G}{ }_{11}-\mathrm{Tz}$ ) imaging involve following steps: (1) Atezolizumab-TCO was first injected into the bloodstream. (2) Atezolizumab-TCO was binded specifically to PD-L1 overexpressed by tumor and concomitantly was cleared from the blood slowly. (3) Then ${ }^{99 m}$ Tc-HYNIC-PEG 11 -Tz was injected. (4) ${ }^{99 m}$ Tc-HYNIC-PEG $_{11}$-Tz was binded to pretargeted Atezolizumab-TCO via in vivo click ligation of the two components, followed by the rapid clearance of the excess Tz-radioligand. The pretargeted imaging strategy clearly delineated PD-L1 expressing H1975 human lung cancer xenografts with high imaging contrast and significantly reduced background radiation dose to nontarget organs. However, the surplus unclicked ${ }^{99 m} \mathrm{Tc}-\mathrm{HYNIC}-\mathrm{PEG}_{11}-\mathrm{Tz}$ is cleared somewhat sluggishly through the intestines. This is, of course, not an ideal situation for imaging abdominal tumor, especially for CRC. Therefore, we will further try to develop a novel Tz-radioligand with more favorable pharmacokinetic profile.

We will add a polypeptide chain containing hydrophilic amino acids between the HYNIC and Tz to shift the excretion of the tetrazine to the renal system, thereby reducing abdominal uptake background and facilitating imaging abdominal tumors. Ultimately, we developed another pretargeted imaging strategy based on the combination of Cetuximab-TCO and ${ }^{99 m}$ Tc-HYNIC-Polypeptide-PEG 11 -Tz for EGFR expression of CRC due to the renal excretion of the novel Tz-radioligand, which further broaden the application scope of pretarged imaging strategy.

\section{Materials And Methods}

\subsection{Reagents and materials}


All reagents and solvents were obtained from commercial sources (Sigma-Aldrich, Peprotech, Merda, Thermo, BD Bioscience, Invitrogen, Millipore, Nest, Hyclone, and Corning) and used as received without further purification. Cetuximab solutions were obtained from Selleck Chemicals. Amine-reactive TCO-NHS was purchased from Click Chemistry Tools. 3-(4-Benzylamino)-1,2,4,5-tetrazine ( $\mathrm{Tz}$ ) bearing an aminereactive linker and O-(2-aminoethyl)-O'-[2-(boc-amino)ethyl]-decaethylene glycol $\left(\mathrm{NH}_{2}-\mathrm{PEG}_{11}-\mathrm{NHBoc}\right)$ were purchased from Xi'an KaiXin Biological. $\mathrm{Na}\left[{ }^{99 \mathrm{~m}} \mathrm{Tc}_{\mathrm{T}}\right] \mathrm{TcO}_{4}$ was purchased from Shanghai Atom Kexing Pharmaceutical Co., Ltd. All solvents used for HPLC analysis within this project were HPLC grade.

\section{2 mAb-TCO modification and characterization}

$0.2 \mathrm{M} \mathrm{NaHCO}_{3}$ was added to Cetuximab in PBS solution (817ul, $12.12 \mathrm{mg} / \mathrm{ml}$ ) to make the final buffer $\mathrm{pH}$ as 8.6. To this solution was added an appropriate volume of TCO-NHS in N,N-dimethylacetamide to to make final protein concentration as $3.0 \mathrm{mg} / \mathrm{ml}, \mathrm{N}, \mathrm{N}$-dimethylacetamide content as $10 \%$, and $\mathrm{TCO} / \mathrm{mAb}$ reaction stoichiometry as 35:1. The resulting solution was incubated with gentle shaking for $3 \mathrm{~h}$ at room temperature in dark. Subsequently, a $40 \mathrm{KD}$ spin desalting column was used for the buffer exchange and purification of the final product. Aggregation and purity of the product were determined with SEC-HPLC. Protein concentration of this product was determined by spectrophotometer at the $280 \mathrm{~nm}$ absorbance. The final product was stored in PBS $(\mathrm{pH}=7.4)$.

3.2ul N,N-Dimethylformamide (DMF) and $2.8 \mathrm{ul} 8 \mathrm{mg} / \mathrm{ml} \mathrm{HYNIC-Polypeptide-PEG_{11 }}$-Tz solution in DMF were added to $24 \mathrm{ul}$ of the product obtained in above step, resulting in a total volume of $30 \mathrm{ul}$ and a

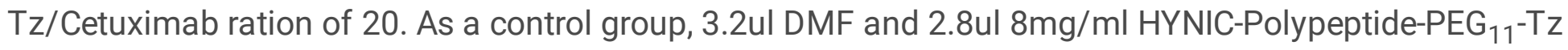
solution in DMF were added to 24ul PBS solution to make the total volume of the reaction solution to be 30ul. The reaction solution was incubated with gentle shaking at $37^{\circ} \mathrm{C}$ for 20 minutes in dark, and then the consumption of HYNIC-Polypeptide-PEG $11_{1}-\mathrm{Tz}$ was determined with RP-HPLC to calculate the effective drug-to-antibody ratio (DAR).

\subsection{Synthesis of Tz derivatives, radiolabelling with $99 \mathrm{mTc}$ and in vitro stability assay}

We designed the chemical structures of HYNIC-PEG $11-T z$ and HYNIC-Polypeptide-PEG $11-T z$, as indicated in Figure 1. Detailed synthesis route procedures are described in our previous work [17] and supplementary material. HYNIC-PEG 11 -Tz and HYNIC-Polypeptide-PEG $11^{-}$Tz was labeled with ${ }^{99 m}$ Tc using tricine as coligand. Briefly, $185 \mathrm{MBq}$ of $\mathrm{Na}\left[{ }^{99 \mathrm{~m}} \mathrm{Tc}\right] \mathrm{TcO}_{4}$ (up to $100 \mu \mathrm{l}$ ) was added to a mixed solution containing 20 ug HYNIC-PEG ${ }_{11}$-Tz or 40 ug HYNIC-Polypeptide-PEG $11^{-T z}, 200 \mu l$ of tricine solution in water $(100 \mathrm{mg} / \mathrm{ml}), 1 \mu \mathrm{l}$ of freshly prepared solution of $\mathrm{SnCl}_{2}\left(1 \mathrm{mg} / \mathrm{ml}\right.$ in $\left.10^{-3} \mathrm{M} \mathrm{HCl}\right)$. The $\mathrm{pH}$ of the mixture was adjusted to 5.0 with $\mathrm{HCl}$. After gently vortexing for a while, the reaction mixture was 
incubated at room temperature for $20 \mathrm{~min}$. Radiochemical purity was determined by reversed-phase high performance liquid chromatography (RP-HPLC) using a gradient of $15: 85 \mathrm{MeCN}: \mathrm{H}_{2} \mathrm{O}$ (both with $0.1 \%$ TFA) to $45: 55 \mathrm{MeCN}: \mathrm{H}_{2} \mathrm{O}$ over $20 \mathrm{~min}$. The procedures of in vitro stability assay of HYNIC-Polypeptide$\mathrm{PEG}_{11}$-Tz in NS, PBS orFBS refer to our previous work [17].

\subsection{Distribution coefficient assay}

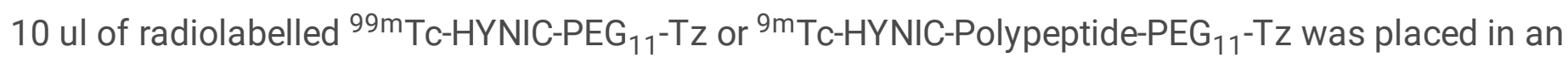
Eppendorf tube containing $500 \mu \mathrm{L}$ of n-octanol and $500 \mu \mathrm{L}$ of PBS $(\mathrm{pH}=7.4)$ solutions. The tube was vortexed vigorously for $1 \mathrm{~min}$ and then centrifuged at $12000 \mathrm{rpm}$ for 5 minutes. The n-octanol/PBS $(\mathrm{pH}=$ 7.4) distribution coefficient was calculated in triplicate for each product. For each replicate, $10 \mu \mathrm{L}$ solution was collected from both phases. Finally, the radioactivity of each fraction was measured in a $y$-counter. The distribution coefficient was calculated as $\log \mathrm{D}(\log \mathrm{D}=\log 10 \mathrm{n}$-octanol counts/aqueous phase counts).

\subsection{In vitro IEDDA between Cetuximab-TCO and 99mTc-HYNIC-Polypeptide-PEG ${ }_{11}$-Tz}

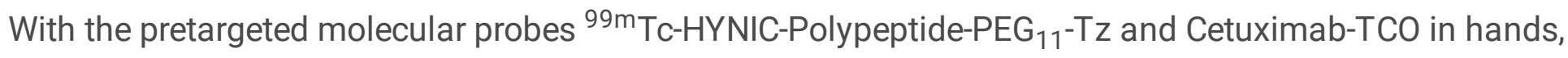
the reactivity of ${ }^{99 \mathrm{~m}} \mathrm{Tc}$-HYNIC-Polypeptide-PEG $11^{-T z}$ towards Cetuximab-TCO was first tested in vitro $(\mathrm{n}=$ 3). Typically, $100 \mu \mathrm{g}$ Cetuximab-TCO was incubated with 8-fold molar equivalents of ${ }^{99 \mathrm{~m} T \mathrm{TC}-\mathrm{HYNIC}-}$ Polypeptide-PEG $11^{-T z}$ at $37^{\circ} \mathrm{C}$ for $30 \mathrm{~min}$. Then, $20 \mu \mathrm{l}$ reaction solution was injected directly into SECHPLC. The fraction of cycloaddition reaction compound ( ${ }^{99} \mathrm{~m}$ Tc-HYNIC-Polypeptide-PEG $11^{-T z} / \mathrm{TCO}-$ Cetuximab) was determined by integrating the radioactive peak corresponding to the ligation compound.

\subsection{Pretargeted cell immunoreactivity binding assay}

HCT116 human CRC cells were obtained from Cell Bank of the Chinese Academy of Sciences. The cells were cultured in RPMI-1640 medium, which was supplemented with $10 \%$ fetal bovine serum and $1 \%$ penicillin-streptomycin. The cells were cultured at $37^{\circ} \mathrm{C}$ in a humidified atmosphere with $5 \% \mathrm{CO}_{2}$. $\mathrm{HCT} 116$ cells were seeded in 24-well plate and grown to $80 \%$ confluence. Experimental group and control group cells were incubated for $2 \mathrm{~h}$ with Cetuximab-TCO $(100 \mathrm{nM})$ and Cetuximab in $500 \mu \mathrm{l} \mathrm{RPMI} 1640$ medium, respectively. Blocking group cells were pretreated by adding an excess of unmodified Cetuximab (100fold) to the wells $2 \mathrm{~h}$ prior to incubation with Cetuximab-TCO. Then, the cells were washed with PBS three times and incubated for $30 \mathrm{~min}$ with ${ }^{99 \mathrm{~m}} \mathrm{Tc}$-HYNIC-Polypeptide-PEG $11^{-\mathrm{Tz}}(0.1 \mu \mathrm{g}, 4.63 \mathrm{MBq} / \mu \mathrm{g})$ in $200 \mu \mathrm{l}$ PBS. After the incubation period, the cells were washed with PBS three times and then lysed with aqueous 
$\mathrm{NaOH}(2 \mathrm{M})$. The membrane-bound activity of each group was determined by measuring the radioactivity of lysed cells using a $\mathrm{Y}$-counter.

\subsection{In vivo pharmacokinetics, biodistribution and imaging of ${ }^{99 m T c-H Y N I C-P o l y p e p t i d e-P E G}{ }_{11}-\mathrm{Tz}$}

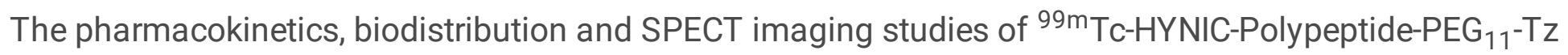
alone were performed to investigate its pharmacokinetic profile, organ biodistriution and excretion pathway. Animal care and experimental procedures were approved by the Animals Ethics Committee of Zhongshan Hospital, Fudan University. For pharmacokinetics study, tumor-bearing mice $(n=3)$ were injected intravenously with ${ }^{99 \mathrm{~m}} \mathrm{Tc}-\mathrm{HYNIC}$-Polypeptide-PEG ${ }_{11}$-Tz and then blood from the tail vein was collected at serial time points $(1,3,5,7,10,15,30$ and $60 \mathrm{~min})$. The blood samples were weighed and measured in a $\mathrm{Y}$-counter along with standards to determine the $\% \mathrm{ID} / \mathrm{g}$.

For biodistribution and imaging studies, male athymic nude mice bearing subcutaneous HCT116 (right shoulder) xenografts were administered ${ }^{99 \mathrm{~m}}{ }^{\mathrm{Tc}}$-HYNIC-Polypeptide-PEG $11^{-T z}(450-500 \mu \mathrm{Ci}$ in $100 \mu \mathrm{l} 0.9 \%$ sterile saline) via intravenous tail vein. Micro-SPECT/CT scans were conducted on the Nano SPECT/CT scanner (BioScan) at $30 \mathrm{~min}, 2 \mathrm{~h}$, and $6 \mathrm{~h}$ post Tz-radioligand injection. Anesthesia and SPECT scan methods refer to our previous work [17]. Biodistribution experiments were performed at the same time point post Tz-radioligand injection with SPECT imaging. The mice were anesthetized with isoflurane and euthanized by cervical dislocation. Blood was withdrawn by heart puncture, and each organ and tissue of interest was harvested, blotted dry, and weighed. The radioactivity of each sample was measured using a $\mathrm{Y}$-counter along with standards to determine the \%ID/g for each sample of interest.

\subsection{Pretargeted biodistribution study}

Male athymic nude mice bearing subcutaneous HCT116 xenografts were administered $100 \mu \mathrm{g}$ Cetuximab-TCO in $100 \mu$ PBS via intravenous tail vein injection. In order to validate the optimal pretargeted interval time, three different accumulation intervals of $24 \mathrm{~h}, 48 \mathrm{~h}$, and $72 \mathrm{~h}$ were performed in the pretargeted biodistribution study $(n=3)$. After three pretargeted accumulation periods, the same mice were then administered ${ }^{99 m}$ Tc-HYNIC-Polypeptide-PEG $11^{-T z}$ (450-500 $\mu \mathrm{Ci}$ in $100 \mu \mathrm{l} 0.9 \%$ sterile saline), also via tail vein injection. Six hours after the Tz-radioligand administration, the animals were anesthetized with isoflurane and blood was withdrawn by heart puncture. Then the mice were euthanized by cervical dislocation, and tissue samples including tumor, heart, lung, liver, spleen, stomach, small intestine, large intestine, kidney, muscle, bone, skin, brain, thyroid, faces (in distal colon), and urine were harvested, blotted dry, and weighed. The radioactivity of all samples was measured in a $\gamma$-counter to determine the $\% \mathrm{ID} / \mathrm{g}$. 


\subsection{Pretargeted biodistribution and imaging comparison}

Nude mice bearing subcutaneous HCT116 xenografts were administered $100 \mu$ g Cetuximab-TCO via intravenous tail vein injection. After an optimal accumulation interval (the highest tumor/blood ratio) of $48 \mathrm{~h}$ according to the results of pretargeted biodistribution study, the mice were then administered ${ }^{99 \mathrm{~m}} \mathrm{Tc}$ HYNIC-PEG $_{11}$-Tz or ${ }^{99 m}$ Tc-HYNIC-Polypeptide-PEG 11 -Tz (450-500 $\mu \mathrm{Ci}$ in $100 \mu \mathrm{l} 0.9 \%$ sterile saline) intravenously. Biodistribution experiments and micro-SPECT/CT scans were conducted at $6 \mathrm{~h}$ post ${ }^{99 \mathrm{~m} T c-}$ HYNIC-PEG 11 -Tz or ${ }^{99 m}$ TC-HYNIC-Polypeptide-PEG 11 -Tz injection.

\section{Results}

\subsection{Synthesis, characterization, radionuclide labeling, radiochemical purity, and in vitro reactivity of molecular probes}

The reaction of Cetuximab modified by TCO-NHS ester was conducted in an alkaline environment $(\mathrm{pH}=$ 8.6) for $3 \mathrm{~h}$. Then Cetuximab-TCO was purified by a $40 \mathrm{KD}$ spin desalting column. The aggregation and purity of the product were $0.73 \%$ and $99.27 \%$ as shown by SEC-HPLC (Supplementary Figure 1 ). After the reaction of purified Cetuximab-TCO with 20-fold molar equivalents of HYNIC-Polypeptide-PEG ${ }_{11}$-Tz was completed, the consumption of HYNIC-Polypeptide-PEG ${ }_{11}$-Tz was used for calculating the average number of TCO conjugated to each Cetuximab. As shown in Supplementary Figure 2, the peak (Rt = 9.3min) at red and blue curve was the ultraviolet (UV) absorption peak of unclicked HYNIC-Polypeptide$\mathrm{PEG}_{11}-\mathrm{Tz}$ at $280 \mathrm{~nm}$ in the control group and reaction group, respectively. The area under peak $(\mathrm{Rt}=$ $9.3 \mathrm{~min}$ ) at the blue curve accounts for $59.5 \%$ of the corresponding red curve peak, indicating that HYNICPolypeptide-PEG 11 -Tz consumed in reaction group accounted for $40.5 \%$. Thus, the average reactive TCO moieties attached to each Cetuximab was $20 \times 40.5 \%=8.1$.

The HYNIC-Polypeptide-PEG ${ }_{11}$-Tz was synthesized mainly from a polypeptide chain (Gly-Arg-Glu-Arg-GluLys) and three available building blocks: 3-(4-benzylamino)-1,2,4,5-tetrazine (Tz) bearing an aminereactive linker, O-(2-aminoethyl)-O'-[2-(boc-amino)ethyl]-decaethylene glycol $\left(\mathrm{NH}_{2}-\mathrm{PEG}_{11}-\mathrm{NHBoc}\right.$ ), and 6hydrazinonicotinyl (HYNIC) bearing a trifluoroacetyl protection group. The polypeptide chain was synthesized throughout Fmoc peptide solid phase synthesis method. Finally, the residue was purified by prep-HPLC (basic condition) to give HYNIC-Polypeptide-PEG ${ }_{11}$-Tz as a red solid in a high purity (95.5\%). HYNIC-Polypeptide-PEG ${ }_{11}$-Tz was radiolabeled with ${ }^{99 \mathrm{~m}} \mathrm{Tc}$ in a specific activity of $4.63 \mathrm{MBq} / \mu \mathrm{g}$ and a radiochemical purity of above $95 \%$ as confirmed by RP-HPLC. 
The in vitro stability of ${ }^{99 \mathrm{~m}} \mathrm{Tc}-\mathrm{HYNIC}$-Polypeptide-PEG 11 -Tz was tested by incubation in NS, PBS, and FBS at $37^{\circ} \mathrm{C}$ and subsequent HPLC analysis. As indicated in Supplementary Figure 3 , the Tz-radioligand remained relatively stable in the three media at early time point (generally above $85 \%$ radiochemical integrity in the three media after $2 \mathrm{~h}$ incubation). With time, however, decomposition of ${ }^{99 \mathrm{~m}} \mathrm{Tc}-\mathrm{HYNIC}$ Polypeptide-PEG 11 -Tz becomes apparent, especially in FBS (only $72.93 \pm 2.51 \%$ and $63.10 \pm 1.77 \%$ of the Tz-radioligand remained intact after $4 \mathrm{~h}$ and $8 \mathrm{~h}$ incubation, respectively). The in vitro stability of ${ }^{99 \mathrm{~m}} \mathrm{Tc}-$ HYNIC-Polypeptide-PEG $11^{-T z}$ was similar to that of ${ }^{99 m}{ }^{\text {Tc-HYNIC-PEG }} 11^{-T z}$ [17].

The n-octanol/PBS ( $\mathrm{pH}=7.4$ ) distribution coefficient assay of ${ }^{99 \mathrm{~m}} \mathrm{Tc}-\mathrm{HYNIC}-\mathrm{PEG}_{11}$-Tz and ${ }^{{ }^{99} \mathrm{~m}} \mathrm{Tc}-\mathrm{HYNIC}$ Polypeptide-PEG ${ }_{11}$-Tz was performed to determine the effect of polypeptide modification on their hydrophilcity. ${ }^{99 \mathrm{~m}} \mathrm{Tc}-\mathrm{HYNIC}-$ Polypeptide-PEG 11 -Tz showed a higher hydrophilic parameter with a logD of $-4.64 \pm 0.21$, while a value of $-3.75 \pm 0.08$ was observed for ${ }^{99 \mathrm{~m}} \mathrm{TC}_{\mathrm{C}}-\mathrm{HYNIC}-\mathrm{PEG}_{11}$-Tz. The incorporation of the hydrophilic polypeptide chain resulted in a more favorable distribution coefficient value, probably due to the charged and hydrophilic amino acid residue side chains present in the linker.

The in vitro reactivity of ${ }^{99 \mathrm{~m}} \mathrm{Tc}$-HYNIC-Polypeptide-PEG ${ }_{11}$-Tz towards Cetuximab-TCO was tested in PBS at $37{ }^{\circ} \mathrm{C}$ for $30 \mathrm{~min}$. Subsequently, a radio-SEC-HPLC analysis was performed. SEC-HPLC showed that the cycloaddition reaction between ${ }^{99 \mathrm{~m}} \mathrm{Tc}-\mathrm{HYNIC}-$ Polypeptide-PEG ${ }_{11}$-Tz and Cetuximab-TCO proceeded near completely, with the 8:1 Tz-to-mAb reaction stoichiometry providing an average yield of $87.83 \pm 3.27 \%$ (Figure 2).

\subsection{Pretargeted HCT116 cell immunoreactivity binding assay}

Pretargeted cell immunoreactivity binding assay was performed using high EGFR-expressing HCT116 cell line. Pretargeted experimental group showed a high radioactivity retention in cells with an average counts per minute (CPM) value of $19,045 \pm 557$ (Figure 3 ). The control and blocking group cells showed a significantly lower activity with an average CPM value of $323 \pm 52$ and $2326 \pm 341$, respectively. The pretargeted cell immunoreactivity binding assay confirmed the specific binding of Cetuximab-TCO to HCT 116 cells and verified preliminarily the feasibility of the molecular probes used for in vivo pretargeted imaging.

\subsection{Molecular probe in vivo experiments}

The pharmacokinetics, biodistribution and SPECT imaging experiments of ${ }^{99 m}$ Tc-HYNIC-Polypeptide$\mathrm{PEG}_{11}-\mathrm{Tz}$ alone were first performed to investigate its pharmacokinetic profile, tissue biodistribution and excretion pathway. The Tz-radioligand was cleared quickly from the circulation and distributed rapidly 
into various tissues and organs. The half-life $\left(t_{1 / 2}\right)$ of blood distribution is $15.72 \pm 1.83 \mathrm{~min}$ (Supplementary Figure 4 ). The short blood $t_{1 / 2}$ ensures low radioactivity exposure to healthy organs and the background signal is relatively low when used for in vivo imaging. For biodistribution and imaging studies of ${ }^{99 \mathrm{~m}} \mathrm{Tc}$-HYNIC-Polypeptide-PEG 11 -Tz alone, biodistribution experiment and Micro-SPECT/CT scans were conducted at $30 \mathrm{~min}, 2 \mathrm{~h}$, and $6 \mathrm{~h}$ post the Tz-radioligand injection. Contrast to the hepatobiliary elimination pathway of ${ }^{99 \mathrm{~m}} \mathrm{Tc}-\mathrm{HYNIC}-\mathrm{PEG}_{11}$-Tz [17], ${ }^{99 \mathrm{~m}} \mathrm{Tc}-\mathrm{HYNIC}-\mathrm{Polypeptide-PEG}_{11} \mathrm{Tz}$ was cleared completely through the urinary system, as indicated in Supplementary Table 1 and Figure 4. The radioactivity in the kidneys was $4.836 \pm 1.887 \% \mathrm{ID} / \mathrm{g}, 4.591 \pm 0.820 \% \mathrm{ID} / \mathrm{g}$, and $2.979 \pm 1.124 \% \mathrm{ID} / \mathrm{g}$ at $30 \mathrm{~min}, 2 \mathrm{~h}$, and $6 \mathrm{~h}$ post the Tz-radioligand injection, respectively. The Tz-radioligand was excreted quickly into bladder and the \%ID/g of urine was $59.010 \pm 36.709,21.112 \pm 23.803$, and $4.212 \pm 3.157$ at 30 min, $2 \mathrm{~h}$, and $6 \mathrm{~h}$ post the Tz-radioligand injection, respectively. The accumulation and retention of the radiotracer in liver, intestinal, and feces remained very low $(<0.3 \% \mathrm{ID} / \mathrm{g}$ at various time points post the Tzradioligand injection), indicating no hepatobiliary elimination pathway for the radioligand. The low uptake $(<0.2 \% \mathrm{ID} / \mathrm{g})$ in $\mathrm{HCT} 116$ tumor at all time points illustrated that the Tz-radioligand showed nonspecific binding to high EGFR-expressing HCT116 tumor. Critically, the accumulations in the thyroid and stomach were particularly low $(<0.3 \% \mathrm{ID} / \mathrm{g})$, suggesting the high in vivo stability of the Tzradioligand. The SPECT imaging at each time point clearly visualized the biodistribution of ${ }^{99 \mathrm{~m}} \mathrm{TC}-\mathrm{HYNIC}$ Polypeptide-PEG 11 -Tz in nude mice bearing subcutaneous HCT116 xenografts (Figure 5).

In order to validate the optimal pretargeted interval time, three different Cetuximab-TCO accumulation intervals of $24 \mathrm{~h}, 48 \mathrm{~h}$, and $72 \mathrm{~h}$ were performed in the pretargeted biodistribution study (Supplementary Table 2 and Figure 6). Biodistribution experiments demonstrated the accumulation in kidneys and urine was relatively high with a \% ID/g of more than 3.0 at each accumulation interval. After filtering through the kidneys, the radiotracer concentrated in the bladder and finally excreted out of the body through urine. In addition to urinary system, high activity levels were observed in the tumor and blood $(>1 \% \mathrm{ID} / \mathrm{g}$ at each accumulation interval), suggesting a great incidence of in vivo click reactions in the blood in addition to ligations at the tumor. The tumor/blood ratio was $0.83,1.40$, and 1.15 , respectively after allowing $24 \mathrm{~h}, 48$ $\mathrm{h}$, and $72 \mathrm{~h}$ for accumulation of Cetuximab-TCO in HCT116 tumor. Thus, it becomes clear that $48 \mathrm{~h}$ represents an optimal accumulation interval between the administration of mAb-TCO and the subsequent injection of Tz-radioligand. The accumulation and retention of the radiotracer in other organs including hypervascular lung, liver, and spleen remained generally very low, which suggested that the pretargeted strategy could efficiently improve the target/non-target ratio and significantly reduce background radiation dose to nontarget organs.

According to the results of pretargeted biodistribution study, the optimal accumulation interval (48 h) was chosen as the accumulation period of Cetuximab-TCO to compare the pretargeted CetuximabTCO $/{ }^{99 m} \mathrm{Tc}^{-H Y N I C}-\mathrm{PEG}_{11}$-Tz andCetuximab-TCO/ ${ }^{99 \mathrm{~m}} \mathrm{TC}-\mathrm{HYNIC}-$ Polypeptide-PEG 11 -Tzbiodistribution and SPECT imaging (Supplementary Table 3 and Figure 7). As ${ }^{99 m}{ }^{\text {TC-HYNIC-PEG }} 1{ }_{11}$-Tz was cleared rapidly via the hepatobiliary system, the radioactivity in the liver, small intestine, and large intestine was relatively 
low with a \%ID/g of $0.194 \pm 0.108,0.081 \pm 0.132$, and $0.082 \pm 0.156$, respectively at $6 \mathrm{~h}$ post ${ }^{99 \mathrm{~m}} \mathrm{Tc}$-HYNIC$\mathrm{PEG}_{11}$-Tz injection. The amount of activity in faces was relatively high $(28.549 \pm 24.662 \% \mathrm{ID} / \mathrm{g})$, suggesting that the surplus unclicked ${ }^{99 \mathrm{~m}} \mathrm{TC}-\mathrm{HYNIC}-\mathrm{PEG}_{11}$-Tz is cleared rapidly through the intestines into the faces and remained in it. Additionally, a small amount of ${ }^{99 \mathrm{~m}} \mathrm{Tc}-\mathrm{HYNIC}-\mathrm{PEG}_{11}$ - $\mathrm{Tz}$ was cleared through urinary system and the accumulation of urine was $1.196 \pm 1.977 \% \mathrm{ID} / \mathrm{g}$ after injecting the Tz-radioligand for $6 \mathrm{~h}$. Pretargeted Cetuximab-TCO/ ${ }^{99 \mathrm{~m}}$ Tc-HYNIC-Polypeptide-PEG 11 -Tz biodistribution demonstrated that the accumulation in kidneys and urine was relatively high with a \% ID $/ \mathrm{g}$ of $3.421 \pm 0.606$ and $21.981 \pm 7.181 \% \mathrm{ID} / \mathrm{g}$, respectively. The uptake levels in liver, intestines, and faces were all less more 0.4 $\% \mathrm{ID} / \mathrm{g}$, indicating that the surplus unclicked ${ }^{99 \mathrm{~m}} \mathrm{Tc}-\mathrm{HYNIC}$-Polypeptide- $\mathrm{PEG}_{11}$-Tz is cleared through urinary system. The tumor/blood ratio was 1.61 and 1.40, respectively for pretargeted Cetuximab-TCO/ ${ }^{99 \mathrm{~m} T c-}$ HYNIC-PEG 11 -Tz and Cetuximab-TCO/ ${ }^{99 \mathrm{~m}}$ Tc-HYNIC-Polypeptide-PEG ${ }_{11}$-Tz biodistribution experiment. Both pretargeted Cetuximab-TCO/ ${ }^{99} \mathrm{~m}$ Tc-HYNIC-PEG 11 -Tz and Cetuximab-TCO $/{ }^{99} \mathrm{~m}$ Tc-HYNIC-Polypeptide$\mathrm{PEG}_{11}$-Tz imagings delineated the HCT116 tumor clearly and the imaging results were consistent with the results of biodistribution, as indicated in Figure 8. Pretargeted Cetuximab-TCO $/{ }^{99 \mathrm{~m}} \mathrm{Tc}-\mathrm{HYNIC}$-Polypeptide$\mathrm{PEG}_{11}$-Tz imaging avoided the retention of the surplus unclicked Tz-radioligand in faces, which favors to specific targeting imaging for CRC.

\section{Discussion}

The pretargeted strategy (Atezolizumab-TCO/ ${ }^{99} \mathrm{~m} T \mathrm{Tc}-\mathrm{HYNIC}_{\mathrm{PEG}} \mathrm{P}_{11} \mathrm{Tz}$ ) that we previously developed could evaluate immune checkpoint ligand PD-L1 expression in tumor and simultaneously reduce the radiation

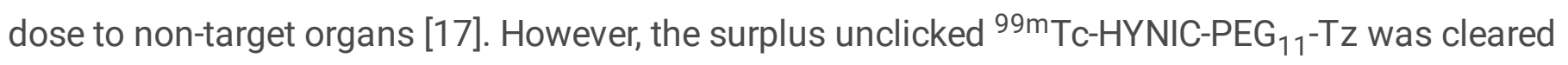
through hepatobiliary system and remained in faces for a long time, which is not favorable for imaging abdominal tumor, especially for CRC. In order to further broaden the application scope of pretarged imaging strategy, we added a polypeptide chain between the HYNIC and Tz to shift the excretion of the Tz-radioligand to the renal system, thereby facilitating imaging CRC.

In order to improve the hydrophilicity of previous ${ }^{99 m}$ Tc-HYNIC-PEG 11 -Tz to facilitate renal excretion, we chose hydrophilic lysine, glutamate, and arginine in designing polypeptide sequence. Ultimately, the polypeptide sequence was determined as Gly-Arg-Glu-Arg-Glu-Lys and was synthesized successfully through the Fmoc-method. In order not to increase the steric resistance of click reaction between Cetuximab-TCO and Tz-radioligand, we added the polypeptide chain between HYNIC and PEG ${ }_{11}$. Finally, the modified Tz-derivative structure was identified as HYNIC-Polypeptide-PEG ${ }_{11}$-Tz. HYNIC-Polypeptide$\mathrm{PEG}_{11}$-Tz was labeled with radionuclide ${ }^{99 \mathrm{~m}} \mathrm{Tc}$ in a similar manner to $\mathrm{HYNIC}-\mathrm{PEG}_{11}$-Tz. The radiochemical purity of final product ${ }^{99 \mathrm{~m}} \mathrm{Tc}$-HYNIC-Polypeptide- $\mathrm{PEG}_{11}$-Tz was more than $95 \%$ and the specific activity

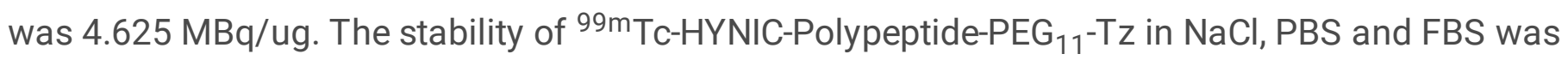
similar to that of ${ }^{99 \mathrm{~m}} \mathrm{Tc}-\mathrm{HYNIC}-\mathrm{PEG}_{11}-\mathrm{Tz}[17]$. 
The urinary excretion of the polypeptide-modified ${ }^{99 \mathrm{~m}} \mathrm{Tc}$-HYNIC-Polypeptide-PEG $\mathrm{F}_{11}$-Tz is probably related to the increased hydrophilicity of the Tz-derivative results from hydrophilic amino acids and the presence of positive charge and lysine residue in the polypeptide chain. It is known that the Tz-scaffold part in the Tz-radioligands is hydrophobic. It is necessary to add hydrophilic structure to improve the hydrophilicity of the final Tz-derivative in the synthetic design of Tz-derivative. In the study conducted by Garcia et al. [18], ${ }^{99 \mathrm{~m}} \mathrm{Tc}-\mathrm{HYNIC}-\mathrm{PEG}_{4}$-Tz-Me was mainly cleared through the hepatobiliary system, and the relative absorption value of the liver and intestine was $9.91 \pm 0.97 \% \mathrm{ID} / \mathrm{g}$ and $23.35 \pm 3.84 \% \mathrm{ID} / \mathrm{g}$, respectively at 1 $\mathrm{h}$ post the Tz-radioligand injection. The authors further added a polypeptide sequence between HYNIC and $\mathrm{PEG}_{4}$ to increase the hydrophilicity of the Tz-radioligand to a $\log \mathrm{D}$ of $-1.05 \pm 0.02$. The newly synthesized Tz-radioligand was excreted mainly through kidneys. The relative absorption value of urine was $81.92 \pm 5.06 \%$ ID at $1 \mathrm{~h}$ post injection. In addition, Nichols et al. [19] and Devaraj et al. [20] successfully synthesized ${ }^{68} \mathrm{Ga}$ and ${ }^{18} \mathrm{~F}$-labeled Tz-coated polymer by introducing a well-established hydrophilic aminodextran backbone into the Tz-derivative, respectively. In our study, the n-octanol/PBS distribution coefficient of ${ }^{99 \mathrm{~m}} \mathrm{Tc}-\mathrm{HYNIC}-\mathrm{PEG}_{11}$-Tz and ${ }^{99 \mathrm{~m}}$ Tc-HYNIC-Polypeptide-PEG ${ }_{11}$-Tz was $-3.75 \pm 0.08$ and 4.64 \pm 0.21 , respectively. The modified ${ }^{99 \mathrm{~m}}$ Tc-HYNIC-Polypeptide- $\mathrm{PEG}_{11}-\mathrm{Tz}$ showed a higher hydrophilic

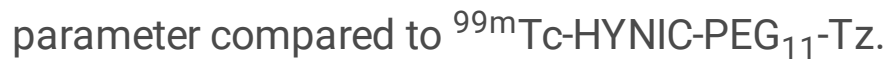

It has been shown that the introduction of positive charge to radiopharmaceuticals can increase their renal clearance and retention [21-23]. Similarly, the introduction of positive charge in the Tz-radioligands increases clearance through the kidneys, whereas Tz derivatives with no charge were mainly excreted via the hepatobiliary system. In the study performed by Zeglis et al., as ${ }^{64} \mathrm{Cu}-\mathrm{NOTA}$-Tz is cleared somewhat sluggishly through the gastrointestinal pathway [24], the authors further created two novel Tz radioligands ( ${ }^{64} \mathrm{Cu}-\mathrm{NOTA}_{-} \mathrm{PEG}_{7}$-Tz and ${ }^{64} \mathrm{Cu}$-SarAr-Tz) in order to improve their pharmacokinetic profiles [25]. For ${ }^{64} \mathrm{Cu}-\mathrm{SarAr}-\mathrm{Tz}$, in addition to the change of the coordination environment from $\mathrm{N}_{3} \mathrm{O}_{3}$ to $\mathrm{N}_{6}$, more importantly, the overall charge of the Tz-radioligand was shifted from -1 to +2 . Finally, the newly synthesized ${ }^{64} \mathrm{Cu}-$ SarAr-Tz eliminates quickly and cleanly through the urinary tract [25]. In a pharmacokinetic profile study including 25 different Tz derivatives radiolabeled with either $A\left[{ }^{18} \mathrm{~F}\right]$ or ${ }^{68} \mathrm{Ga}$, Meyer et al. [26] observed that ${ }^{68} \mathrm{Ga}-\mathrm{NODA}-\mathrm{Tz}$ was excreted through renal pathway and AI[ $\left.{ }^{18} \mathrm{~F}\right]-\mathrm{NODA}-\mathrm{Tz}$ through hepatic and intestinal pathway. The authors suggested that the different excretion pathways of the two Tz-radioligands was resulted from the charge difference between ${ }^{68} \mathrm{Ga}-\mathrm{NODA}-\mathrm{Tz}$ (net charge: +1 ) and AI[ $\left.{ }^{18} \mathrm{~F}\right]-N O D A-T z$ (net charge: 0 ). In addition, the authors found that uncharged Al[ $\left.{ }^{18} \mathrm{~F}\right]-N O T A-l y s i n e-T z$ was excreted mainly through the liver and intestines, while charged AI $\left[{ }^{18} \mathrm{~F}\right]-\mathrm{NOTA}-(\mathrm{lysine}){ }_{2}{ }^{-\mathrm{Tz}}$ (net charge: +1 ) and AI $\left[{ }^{18} \mathrm{~F}\right]-$ NOTA-(lysine) ${ }_{3}$-Tz (net charge: +2 ) were excreted mainly through the kidneys [26]. Besides, it has been reported that renal uptake and excretion may be related to the presence of lysine residues in the molecular structure $[27,28]$.

After investigating three different Cetuximab-TCO accumulation internval of $24 \mathrm{~h}, 48 \mathrm{~h}$, and $72 \mathrm{~h}$, we found that the tumor/blood ratio was highest (48h: $1.40 \mathrm{vs} .24 \mathrm{~h}: 0.83$ and $72 \mathrm{~h}: 1.15)$ for allowing $48 \mathrm{~h}$ 
accumulation of Cetuximab-TCO, which represents an optimal accumulation interval. After injecting Cetuximab-TCO for $24 \mathrm{~h}$, the accumulation of the mAb-TCO at HCT116 tumor had not reached a saturation status due to its large molecular weight and poor tissue infiltration. Simultaneously, there existed a large of circulating Cetuximab-TCOs in the blood since most Cetuximab-TCOs had not been eliminated out of the body, which leads to a great incidence of in vivo click reactions in the blood in addition to ligations at the tumor after ${ }^{99 \mathrm{~m}} \mathrm{Tc}$-HYNIC-Polypeptide-PEG $11^{-}$-Tz injection. In fact, the biodistribution data for $24 \mathrm{~h}$ accumulation showed that the uptake in the blood $(1.596 \pm 0.477 \% \mathrm{ID} / \mathrm{g}) \mathrm{was}$ even higher than that at the tumor $(1.320 \pm 0.141 \% \mathrm{ID} / \mathrm{g})$. Biodistribution data demonstrated that activity level in the blood was relatively lower in the accumulation interval of $48 \mathrm{~h}(1.210 \pm 0.202 \% \mathrm{ID} / \mathrm{g})$ compared to $24 \mathrm{~h}(1.596 \pm 0.477 \% \mathrm{ID} / \mathrm{g})$; and activity level at the tumor was relatively higher in the accumulation interval of $48 \mathrm{~h}(1.688 \pm 0.539 \% \mathrm{ID} / \mathrm{g})$ compared to $24 \mathrm{~h}(1.320 \pm 0.141 \% \mathrm{ID} / \mathrm{g})$. Activity levels of blood and tumor were both lower (Blood: $72 \mathrm{~h}: 1.019 \pm 0.414 \mathrm{vs}$. $48 \mathrm{~h}: 1.210 \pm 0.202 \% \mathrm{ID} / \mathrm{g}$; Tumor: $72 \mathrm{~h}: 1.174 \pm 0.040$ vs. $48 \mathrm{~h}: 1.688 \pm 0.539 \% \mathrm{ID} / \mathrm{g}$ ) in the accumulation interval of $72 \mathrm{~h}$ compared to $48 \mathrm{~h}$. The lower activity level of blood and tumor in the accumulation interval of $72 \mathrm{~h}$ compared to $48 \mathrm{~h}$ was probably resulted from the slow isomerization conversion from TCO into less reactive cis-cyclooctene (CCO) due to the prolonged in vivo period of Cetuximab-TCO. Rossin et al. [29] investigated the deactivation mechanism of mAb-TCO in vivo and found that the TCO-CCO isomerization conversion via copper-containing proteins such as transcuprein, mouse serum albumin, and ceruloplasmin was the sole deactivation pathway of mAb-TCO in serum for a long time. CCO is of 5 orders of magnitude less reactive toward tetrazines than TCO.

According to the results of pretargeted biodistribution study, we chose the optimal accumulation interval $(48 \mathrm{~h})$ to compare the pretargeted Cetuximab-TCO $/{ }^{99 \mathrm{~m}} \mathrm{Tc}-\mathrm{HYNIC}-\mathrm{PEG}_{11}-\mathrm{Tz}$ andCetuximab-TCO $/{ }^{99 \mathrm{~m}} \mathrm{Tc}-$ HYNIC-Polypeptide-PEG 11 -Tzbiodistribution and SPECT imaging using subcutaneous HCT116 xenografts mice. The results demonstrated that the surplus unclicked ${ }^{99 m} \mathrm{Tc}-\mathrm{HYNIC}-\mathrm{PEG}_{11}-\mathrm{Tz}$ is cleared rapidly through the intestines into the faces and remained in it for a long time. The surplus unclicked ${ }^{99 \mathrm{~m}} \mathrm{Tc}$ HYNIC-Polypeptide-PEG ${ }_{11}-\mathrm{Tz}$ is cleared completely through urinary system. The tumor/blood ratio was 1.61 and 1.40, respectively for pretargeted Cetuximab-TCO/ ${ }^{99 m} \mathrm{Tc}^{-H Y N I C-P E G}{ }_{11}$-Tz and Cetuximab-TCO ${ }^{199 \mathrm{~m}} \mathrm{Tc}$-HYNIC-Polypeptide-PEG ${ }_{11}$-Tz biodistribution. Both pretargeted imaging strategies delineated the HCT116 tumor clearly. However, pretargeted Cetuximab-TCO / ${ }^{99 m}$ Tc-HYNIC-Polypeptide-PEG $11^{-T z}$ imaging avoided the retention of the surplus unclicked Tz-radioligand in faces, which favors to specific targeting imaging for CRC. Shi et al. [30] also once used a pretargeted strategy (Cetuximab-Tz or Panitumumab-Tz/AI[ ${ }^{18}$ F]-NOTA-Reppe anhydride) for HCT116 tumor EGFR targeting imaging. In the study conducted by Shi et al., the tumor/blood ratio was $13.02 \pm 0.64$ and $10.15 \pm 1.56$, respectively for pretargeted Cetuximab-Tz and Panitumumab-Tz/AI $\left[{ }^{18} \mathrm{~F}\right]-$ NOTA-Reppe anhydride biodistribution. Both pretargeted imaging strategies delineated the HCT116 tumor clearly. However, due to the fact that Al[ $\left.{ }^{18} \mathrm{~F}\right]-$ NOTA-Reppe anhydride was excreted through hepatobiliary system and remained in faces for a long time, the authors were unable to resolve the problem that imaging agent remained in faces interfered with CRC imaging. 


\section{Conclusion}

We successfully developed a novel pretargeted imaging strategy (Cetuximab-TCO/ ${ }^{99 \mathrm{~m}} \mathrm{Tc}-\mathrm{HYNIC}$ Polypeptide-PEG ${ }_{11}-\mathrm{Tz}$ ) for imaging colorectal cancer since the surplus unclicked ${ }^{99 \mathrm{~m}} \mathrm{Tc}-\mathrm{HYNIC}$ Polypeptide-PEG ${ }_{11}-\mathrm{Tz}$ is cleared through urinary system and produces low abdominal uptake background, which further broadens the application scope of pretargeted imaging strategy.

\section{Declarations}

\section{List of Abbreviations}

ACN Acetonitrile

CT Computed Tomography

CPM Counts per Minute

CRC Colorectal cancer

CCO Cis-cyclooctene

DMEM Dulbecco's Modification of Eagle's Medium Dulbecco

DAR Drug to Antibody Ratio

DMF N,N-Dimethylformamide

EGFR Epidermal Growth Factor Receptor

FBS Fetal Bovine Serum

HYNIC 6-hydrazinonicotinic Acid

IEDDA Inverse Electron Demand [4+2] Diels-Alder cycloaddition

IFN- $\mathrm{Y}$ Interferon- $\mathrm{Y}$

keV Kiloelectron Volt

LC-MS Liquid Chromatography- Mass Spectrometry

MBq Megabecquerel

MRI Magnetic Resonance Imaging

$\mathrm{NaCl}$ Sodium Chloride 
PD-L1 Programmed Cell Death Ligand-1

PBS Phosphate Buffer Saline

RP-HPLCReversed-phase High-performanceLiquid Chromatography

RCP Radiochemical Purity

SEC Size Exclusion Chromatography

SPECT Single Photon Emission Computed Tomography

Tz 1,2,4,5-terazine

TCO Trans-cyslooctene

Tricine N-tris-hydroxymethyl-Methylglycine

${ }^{18}$ F-FDG 2-deoxy-2-[ ${ }^{18}$ F]fluoro-D-glucose

${ }^{99 m}$ Tc Technetium-99m

$\% \mathrm{ID} / \mathrm{g}$ Percentage of the Injected Dose Per Gram of Tissue Mass

\section{Acknowledgments}

We want to thank for the technical supports from Prof. Yingjian Zhang and Dr. Jianping Zhang from Center for Biomedical Imaging, Fudan University, and Shanghai Engineering Research Center of Molecular Imaging Probes.

\section{Authors' contributions}

LQ, DFC and HCS designed the study and take responsibility for the integrity and accuracy of the data analysis. LQ, HT and DFC conceived and designed the experiments; LQ performed the main experiments and analysed the data; QYL, ZS, JZ and TTW helped do analysis and interpretation of data. LQ drafted the manuscript. DFC and HCS revised the manuscript. All authors contributed to this manuscript. All authors read and approved the final manuscript.

\section{Funding}

This study was funded by The National Nature Science Foundation of China $(11875114,81671735$, and 81871407) and Open Large Infrastructure Research of Chinese Academy of Science. 


\section{Availability of data and materials}

All data generated or analysed during this study are included in this published article and its supplementary information files.

\section{Ethics approval and consent to participate}

Animal care and all the procedures of animal experiments performed in this study were approved by the Animals Ethics Committee of Zhongshan Hospital, Fudan University.

\section{Consent for publication}

All authors agree to submit the article for publication.

\section{Competing interests}

The authors declare that they have no competing interests.

\section{References}

[1] Torre L A, Bray F, Siegel R L, et al. Global cancer statistics, 2012[J]. CA Cancer J Clin, 2015,65(2):87108.

[2] DeSantis C E, Lin C C, Mariotto A B, et al. Cancer treatment and survivorship statistics, 2014[J]. CA Cancer J Clin, 2014,64(4):252-271.

[3] van Rijn J C, Reitsma J B, Stoker J, et al. Polyp miss rate determined by tandem colonoscopy: a systematic review[J]. Am J Gastroenterol, 2006,101(2):343-350.

[4] Turker N S, Heidari P, Kucherlapati R, et al. An EGFR targeted PET imaging probe for the detection of colonic adenocarcinomas in the setting of colitis[J]. Theranostics, 2014,4(9):893-903.

[5] Liu J, Zuo X, Li C, et al. In vivo molecular imaging of epidermal growth factor receptor in patients with colorectal neoplasia using confocal laser endomicroscopy[J]. Cancer Lett, 2013,330(2):200-207.

[6] Winkler A M, Rice P F, Weichsel J, et al. In vivo, dual-modality OCT/LIF imaging using a novel VEGF receptor-targeted NIR fluorescent probe in the AOM-treated mouse model[J]. Mol Imaging Biol, 2011,13(6):1173-1182.

[7] Hsiung P L, Hardy J, Friedland S, et al. Detection of colonic dysplasia in vivo using a targeted heptapeptide and confocal microendoscopy[J]. Nat Med, 2008,14(4):454-458. 
[8] Rusckowski M, Gupta S, Liu G, et al. Evidence of specificity of radiolabeled phage display peptides for the TAG-72 antigen[J]. Cancer Biother Radiopharm, 2007,22(4):564-572.

[9] Yang M, Fan Q, Zhang R, et al. Dragon fruit-like biocage as an iron trapping nanoplatform for high efficiency targeted cancer multimodality imaging[J]. Biomaterials, 2015,69:30-37.

[10] Yoon S M, Myung S J, Kim I W, et al. Application of near-infrared fluorescence imaging using a polymeric nanoparticle-based probe for the diagnosis and therapeutic monitoring of colon cancer[J]. Dig Dis Sci, 2011,56(10):3005-3013.

[11] Sartore-Bianchi A, Martini M, Molinari F, et al. PIK3CA mutations in colorectal cancer are associated with clinical resistance to EGFR-targeted monoclonal antibodies[J]. Cancer Res, 2009,69(5):1851-1857.

[12] Aerts H J, Dubois L, Perk L, et al. Disparity between in vivo EGFR expression and 89Zr-labeled cetuximab uptake assessed with PET[J]. J Nucl Med, 2009,50(1):123-131.

[13] Spiegelberg D, Mortensen A C, Selvaraju R K, et al. Molecular imaging of EGFR and CD44v6 for prediction and response monitoring of HSP90 inhibition in an in vivo squamous cell carcinoma model[J]. Eur J Nucl Med Mol Imaging, 2016,43(5):974-982.

[14] Shih Y H, Peng C L, Lee $S$ Y, et al. 111In-cetuximab as a diagnostic agent by accessible epidermal growth factor (EGF) receptor targeting in human metastatic colorectal carcinoma[J]. Oncotarget, 2015,6(18):16601-16610.

[15] Guo Y, Parry J J, Laforest R, et al. The role of p53 in combination radioimmunotherapy with 64CuDOTA-cetuximab and cisplatin in a mouse model of colorectal cancer[J]. J Nucl Med, 2013,54(9):16211629.

[16] Zeng D, Guo Y, White A G, et al. Comparison of conjugation strategies of cross-bridged macrocyclic chelators with cetuximab for copper-64 radiolabeling and PET imaging of EGFR in colorectal tumorbearing mice[J]. Mol Pharm, 2014,11(11):3980-3987.

[17] Qiu L, Tan H, Lin Q, et al. A Pretargeted Imaging Strategy for Immune Checkpoint Ligand PD-L1 Expression in Tumor Based on Bioorthogonal Diels-Alder Click Chemistry[J]. Mol Imaging Biol, 2019.

[18] Garcia M F, Gallazzi F, Junqueira M S, et al. Synthesis of hydrophilic HYNIC-[1,2,4,5]tetrazine conjugates and their use in antibody pretargeting with (99m)Tc[J]. Org Biomol Chem, 2018,16(29):52755285 .

[19] Nichols B, Qin Z, Yang J, et al. 68Ga chelating bioorthogonal tetrazine polymers for the multistep labeling of cancer biomarkers[J]. Chem Commun (Camb), 2014,50(40):5215-5217.

[20] Devaraj N K, Thurber G M, Keliher E J, et al. Reactive polymer enables efficient in vivo bioorthogonal chemistry[J]. Proc Natl Acad Sci U S A, 2012,109(13):4762-4767. 
[21] Garcia G E, Schweinsberg C, Maes V, et al. Influence of the molecular charge on the biodistribution of bombesin analogues labeled with the [99mTc(CO)3]-core[J]. Bioconjug Chem, 2008,19(12):2409-2416.

[22] Tsiapa I, Loudos G, Varvarigou A, et al. Biological evaluation of an ornithine-modified (99m)Tclabeled RGD peptide as an angiogenesis imaging agent[J]. Nucl Med Biol, 2013,40(2):262-272.

[23] Antunes P, Ginj M, Walter M A, et al. Influence of different spacers on the biological profile of a DOTAsomatostatin analogue[J]. Bioconjug Chem, 2007,18(1):84-92.

[24] Zeglis B M, Sevak K K, Reiner T, et al. A pretargeted PET imaging strategy based on bioorthogonal Diels-Alder click chemistry[J]. J Nucl Med, 2013,54(8):1389-1396.

[25] Zeglis B M, Brand C, Abdel-Atti D, et al. Optimization of a Pretargeted Strategy for the PET Imaging of Colorectal Carcinoma via the Modulation of Radioligand Pharmacokinetics[J]. Mol Pharm, 2015,12(10):3575-3587.

[26] Meyer J P, Kozlowski P, Jackson J, et al. Exploring Structural Parameters for Pretargeting Radioligand Optimization[J]. J Med Chem, 2017,60(19):8201-8217.

[27] Janzer M, Larbig G, Kubelbeck A, et al. Drug Conjugation Affects Pharmacokinetics and Specificity of Kidney-Targeted Peptide Carriers[J]. Bioconjug Chem, 2016,27(10):2441-2449.

[28] Flook A M, Yang J, Miao Y. Substitution of the Lys linker with the beta-Ala linker dramatically decreased the renal uptake of $99 \mathrm{mTc}$-labeled Arg-X-Asp-conjugated and X-Ala-Asp-conjugated alphamelanocyte stimulating hormone peptides[J]. J Med Chem, 2014,57(21):9010-9018.

[29] Rossin R, van den Bosch S M, Ten H W, et al. Highly reactive trans-cyclooctene tags with improved stability for Diels-Alder chemistry in living systems[J]. Bioconjug Chem, 2013,24(7):1210-1217.

[30] Shi X, Gao K, Huang H, et al. Pretargeted Immuno-PET Based on Bioorthogonal Chemistry for Imaging EGFR Positive Colorectal Cancer[J]. Bioconjug Chem, 2018,29(2):250-254.

\section{Figures}



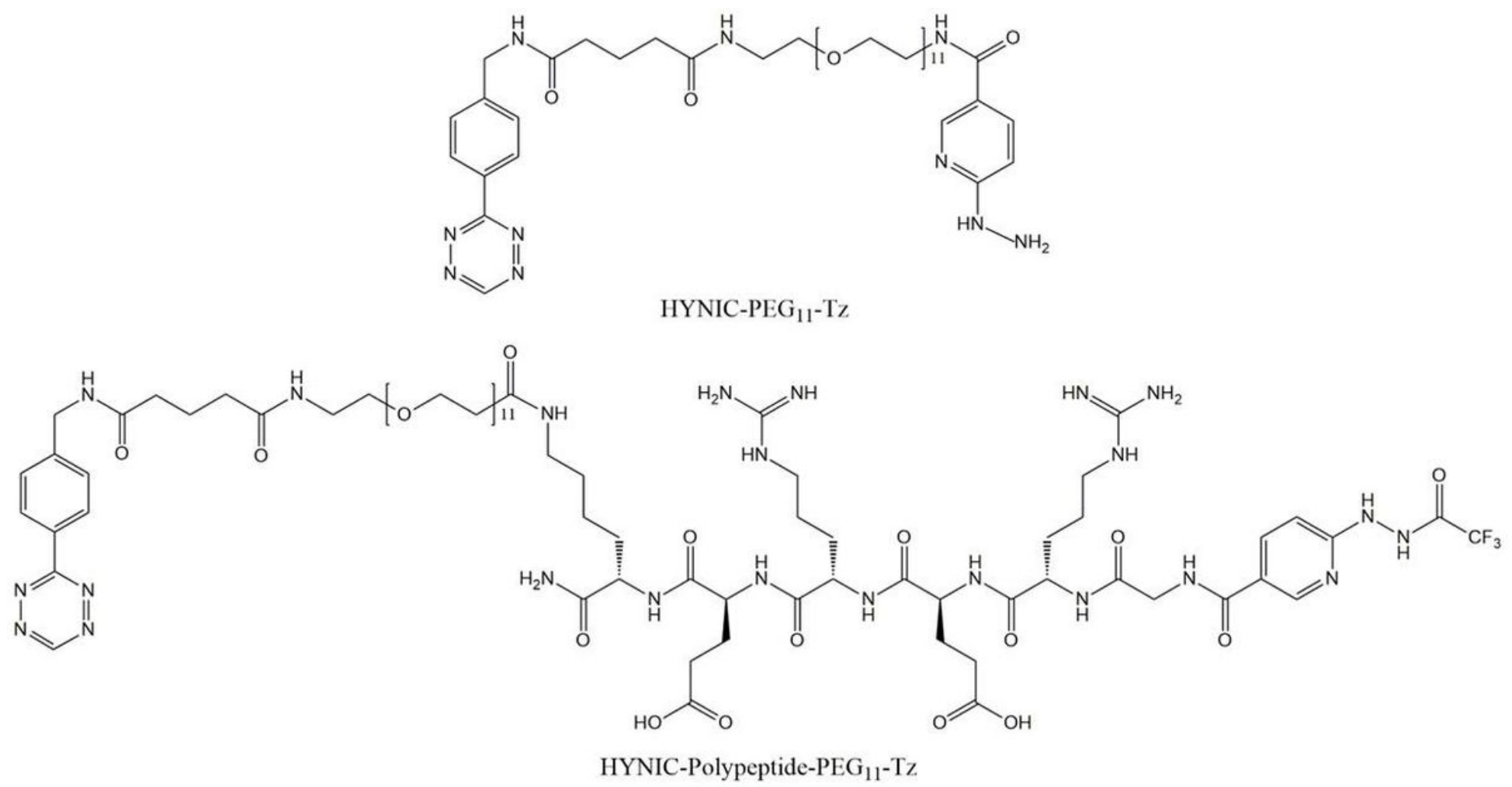

Figure 1

Chemical structures for HYNIC-PEG11-Tz and HYNIC-Polypeptide-PEG11-Tz. 


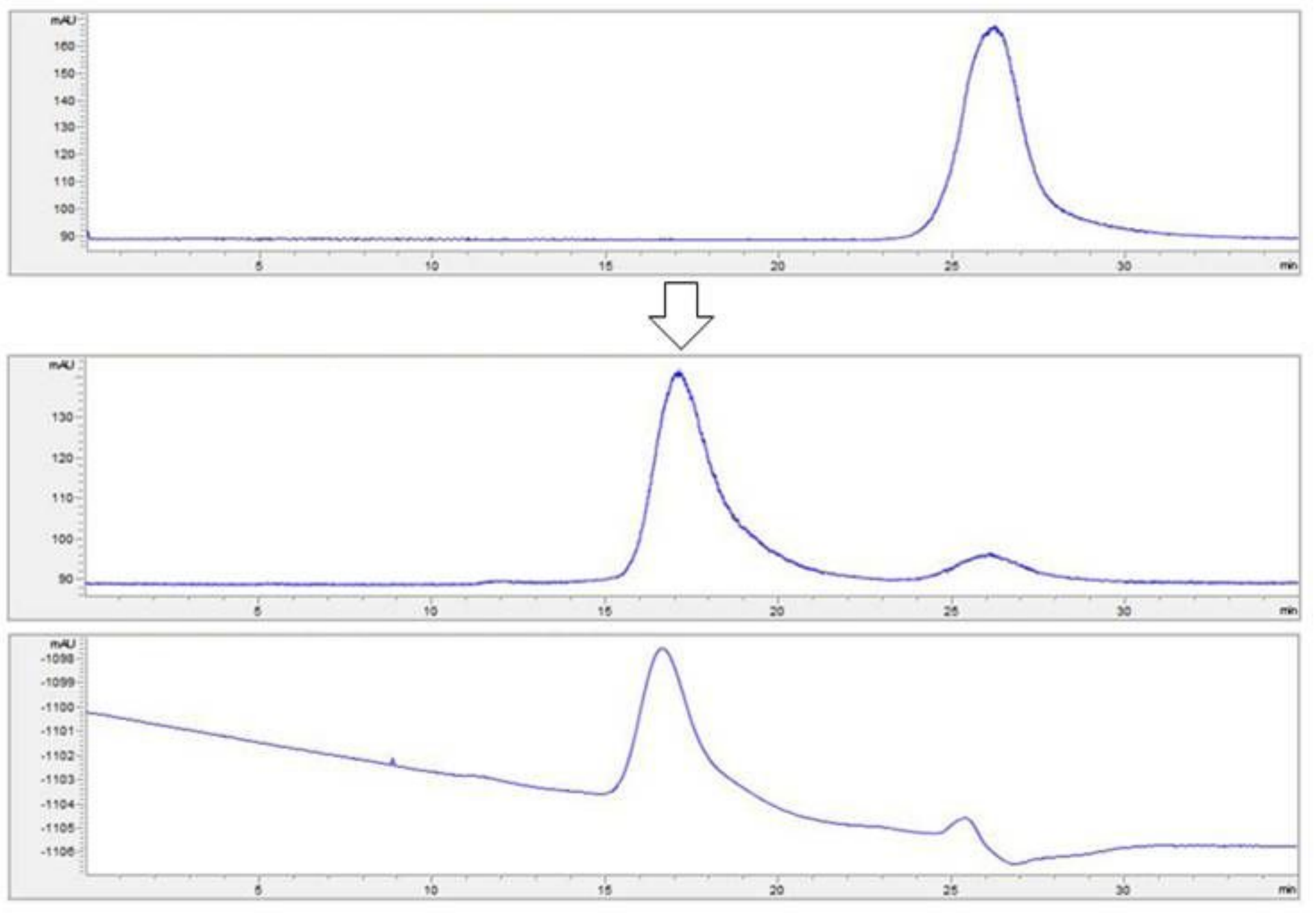

\section{Figure 2}

Top map: The radio-SEC-HPLC result for 99mTC-HYNIC-Polypeptide-PEG11-Tz alone. A peak was observed at the retention time $(\mathrm{Rt})=26.14 \mathrm{~min}$ corresponding to the $99 \mathrm{mTC}$-HYNIC-Polypeptide-PEG11Tz. Middle map: The radio-SEC-HPLC result after incubation with the conjugation between $99 \mathrm{mTC}-\mathrm{HYNIC}-$ Polypeptide-PEG11-Tz and Cetuximab-TCO in PBS for $30 \mathrm{~min}$. The peak observed previously at Rt $=26.14$ min had almost disappeared (The integral of the peak is 11.54\%) concomitant with the appearance of a new peak at Rt $=16.05$ min, indicating the formation of the "99mTc-HYNIC-Polypeptide-PEG11-Tz/TCOCetuximab" complex (The integral of the peak is $88.46 \%$ ). Bottom map: The peak observed at Rt $=16.05$ min and 26.14 min corresponds to the UV absorption peak at $280 \mathrm{~nm}$ for " $99 \mathrm{mTC}$-HYNIC-PolypeptidePEG11-Tz/TCO-Cetuximab" complex and 99mTc-HYNIC-Polypeptide-PEG11-Tz, respectively. 


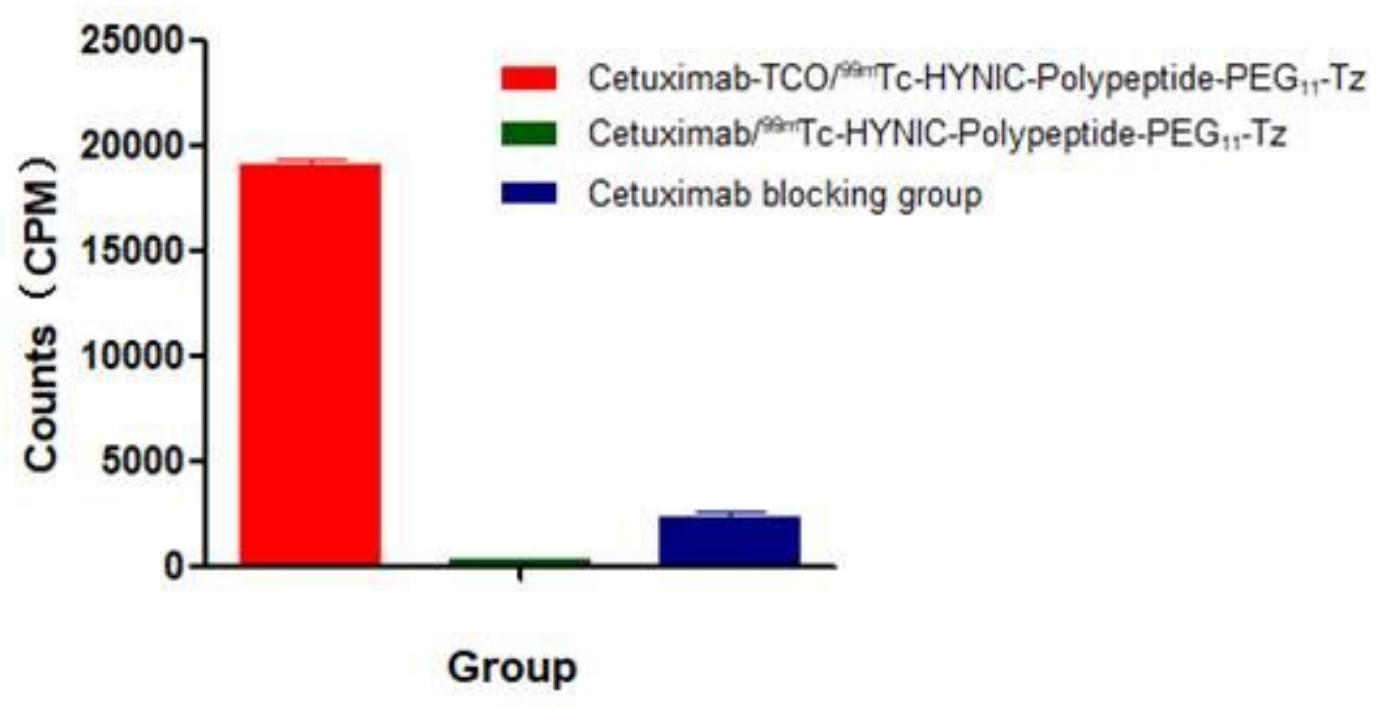

Figure 3

Pretargeted HCT116 Cell Immunoreactivity Binding Assay.

${ }^{99 m}$ TC-HYNIC-Polypeptide-PEG 11 -Tz Biodistribution

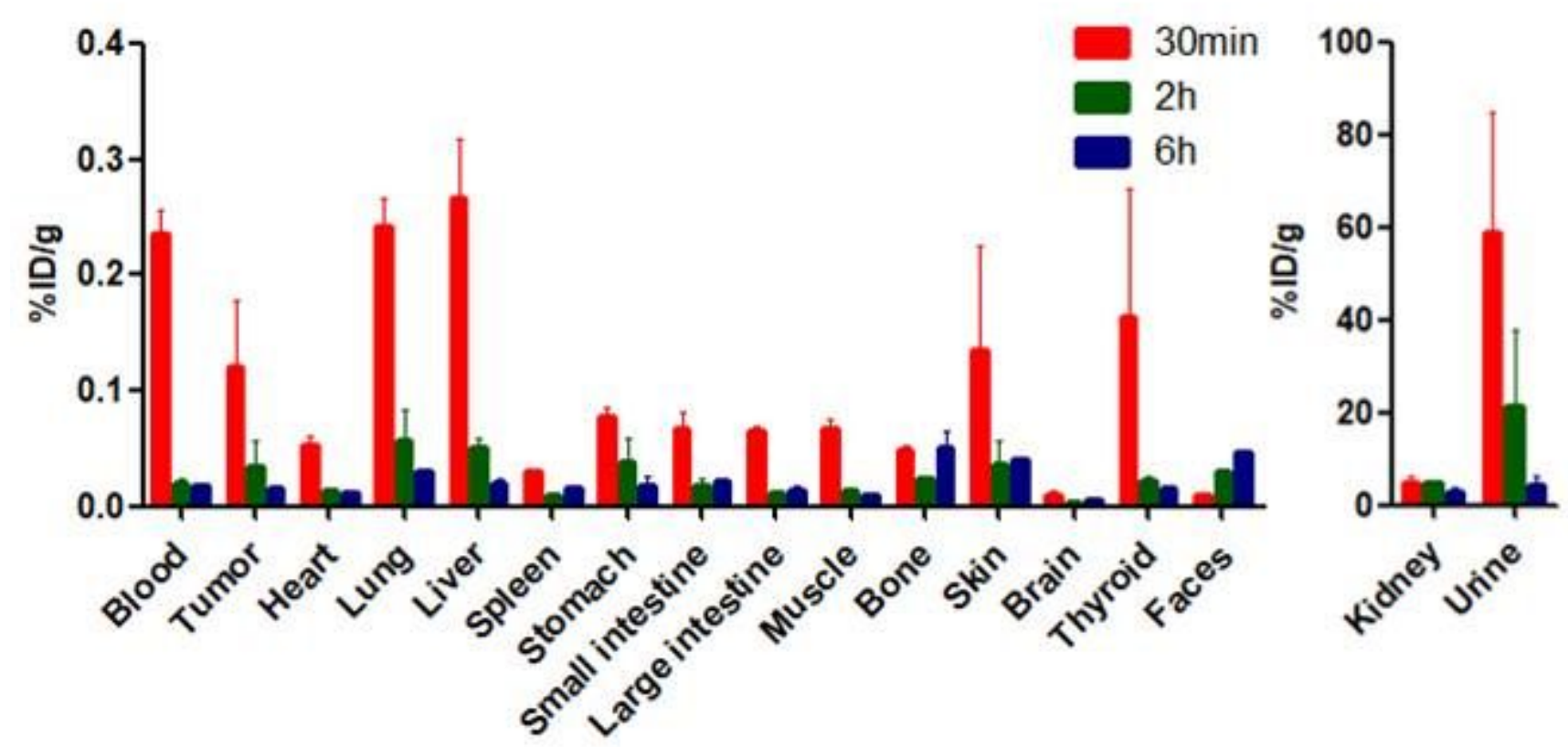

Figure 4

Biodistribution of 99mTc-HYNIC-Polypeptide-PEG11-Tz. Mice bearing HCT116 xenografts were administered 99mTc-HYNIC-Polypeptide-PEG11-Tz via tail vein injection. The mice were euthanized, and tissues of interest were harvested at $30 \mathrm{~min}, 2 \mathrm{~h}$, and $6 \mathrm{~h}$ after the Tz-radioligand injection. 

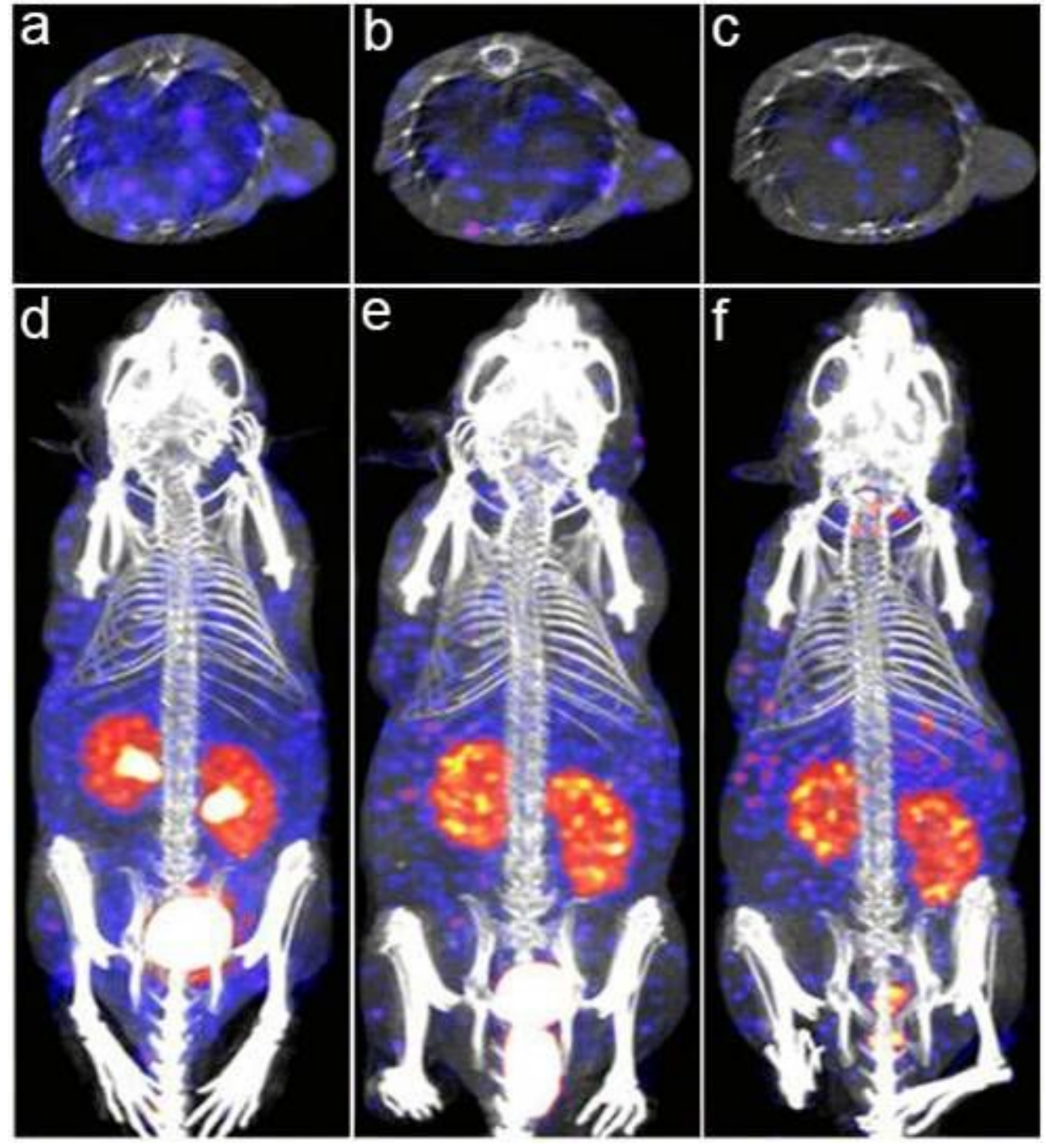

Figure 5

Small-animal SPECT/CT imaging of live mice bearing subcutaneous HCT116 xenografts at 30 min (a and d), $2 \mathrm{~h}$ ( $\mathrm{b}$ and e), and $6 \mathrm{~h}$ ( $\mathrm{c}$ and $\mathrm{f}$ ) after 99mTc-HYNIC-Polypeptide-PEG11-Tz injection. a-c single transverse slices passing through the tumors in $\mathrm{d}-\mathrm{f}$ (posterior projections). 


\section{Pretargeted Biodistribution}

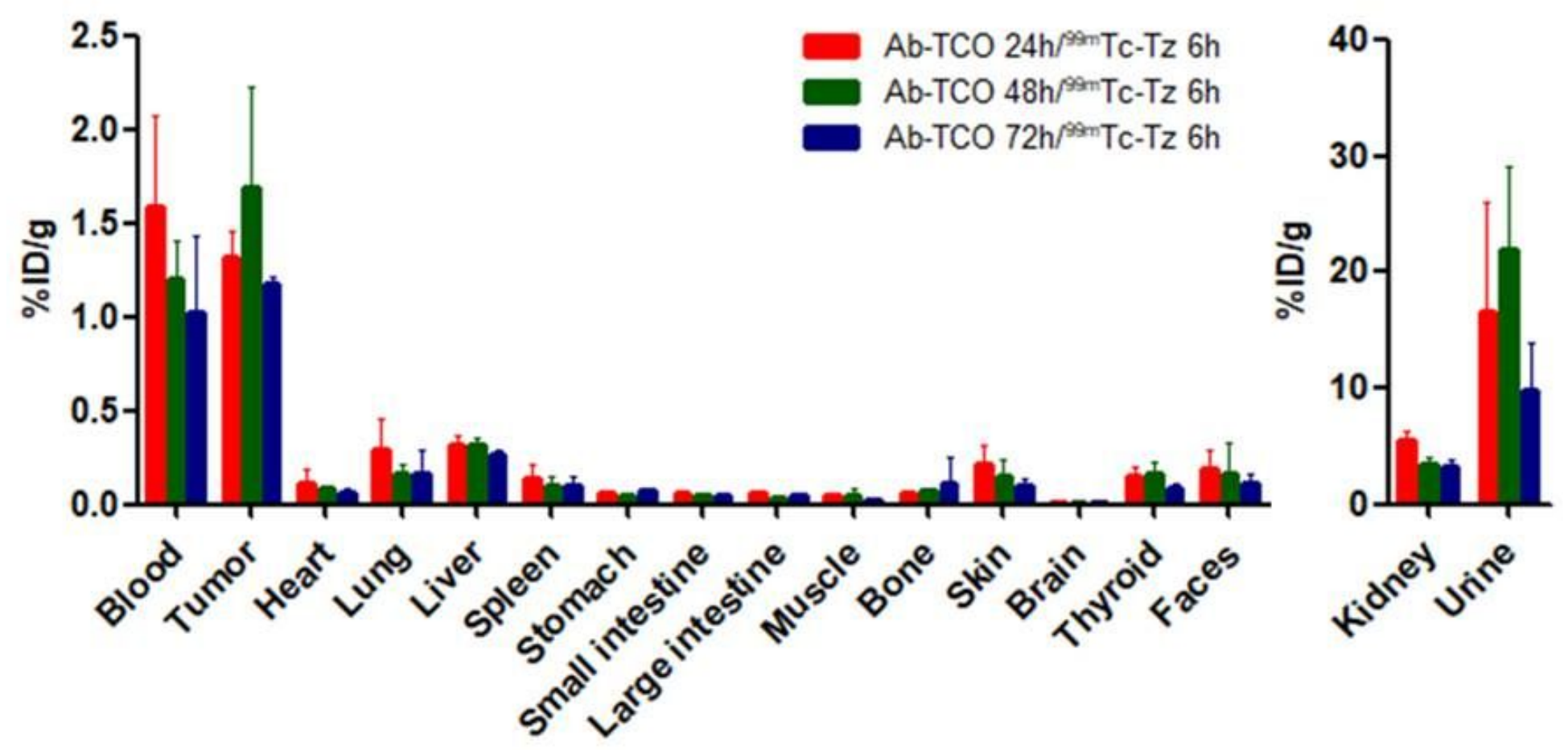

Figure 6

Biodistribution of live mice bearing subcutaneous HCT116 xenografts preinjected with Cetuximab-TCO followed $24 \mathrm{~h}, 48 \mathrm{~h}$, and $72 \mathrm{~h}$ later by $99 \mathrm{mTc}$-HYNIC-Polypeptide-PEG11-Tz. The animals were euthanized, and tissues of interest were harvested at $6 \mathrm{~h}$ after the Tz-radioligand injection. 


\section{Pretargeted Biodistribution Comparison}

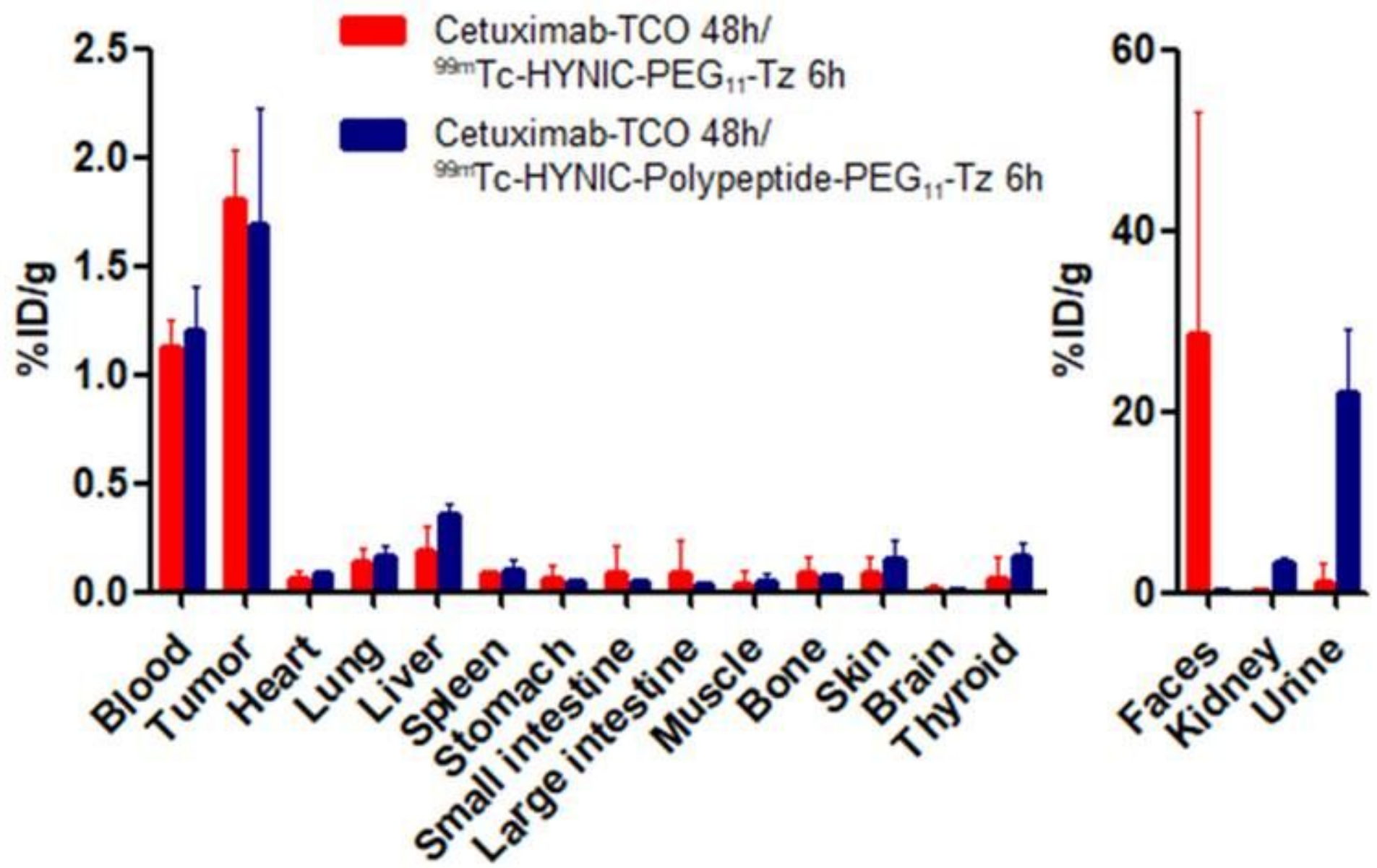

Figure 7

Biodistribution comparision of live mice bearing subcutaneous HCT116 xenografts preinjected with Cetuximab-TCO followed $48 \mathrm{~h}$ later by $99 \mathrm{mTc}$-HYNIC-PEG11-Tz or 99mTc-HYNIC-Polypeptide-PEG11-Tz. The animals were euthanized, and tissues of interest were harvested at $6 \mathrm{~h}$ after $99 \mathrm{mTc}-\mathrm{HYNIC}-\mathrm{PEG} 11-\mathrm{Tz}$ or 99mTc-HYNIC-Polypeptide-PEG11-Tz injection. 


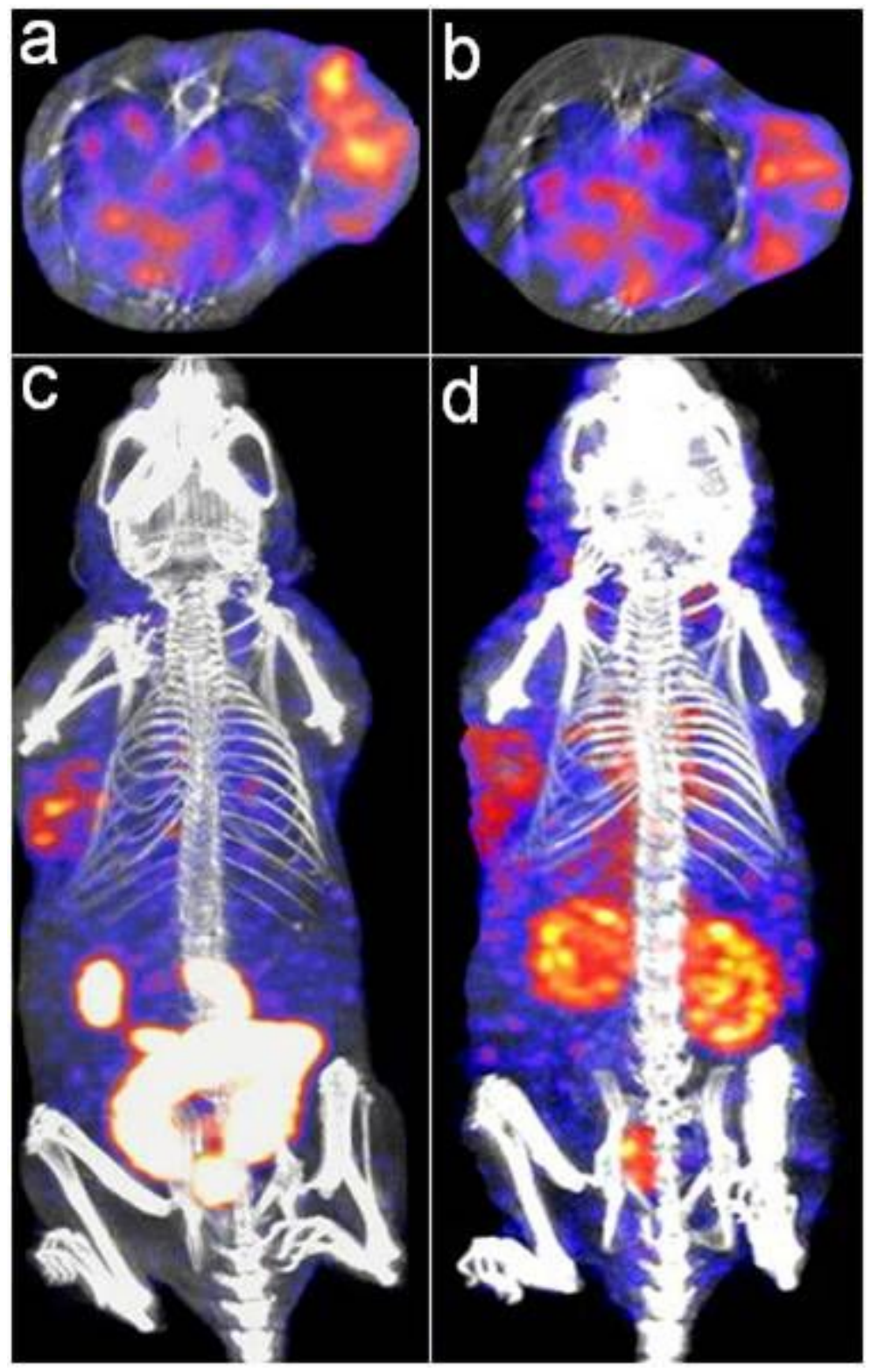

Figure 8

Pretargeted Cetuximab-TCO/99mTc-HYNIC-PEG11-Tz and Cetuximab-TCO /99mTc-HYNIC-PolypeptidePEG11-Tz imaging comparison. Nude mice bearing subcutaneous HCT116 xenografts were administered Cetuximab-TCO $48 \mathrm{~h}$ prior to the injection of 99mTc-HYNIC-PEG11-Tz (a and c) or 99mTc-HYNICPolypeptide-PEG11-Tz ( $b$ and $d$ ) via the tail vein. The images were acquired at $6 \mathrm{~h}$ after $99 \mathrm{mTc}-\mathrm{HYNIC}-$ PEG11-Tz or 99mTc-HYNIC-Polypeptide-PEG11-Tz injection. a-b single transverse slices passing through the tumors in c-d (posterior projections).

\section{Supplementary Files}

This is a list of supplementary files associated with this preprint. Click to download.

- ManuscriptSupplementaryinformation.docx 